Dieses Dokument ist eine Zweitveröffentlichung (Verlagsversion) /

This is a self-archiving document (published version)

„Dieser Beitrag ist mit Zustimmung des Rechteinhabers aufgrund einer (DFG-geförderten) Allianzbzw. Nationallizenz frei zugänglich."

This publication is openly accessible with the permission of the copyright owner. The permission is granted within a nationwide license, supported by the German Research Foundation (abbr. in German DFG).

https://www.nationallizenzen.de
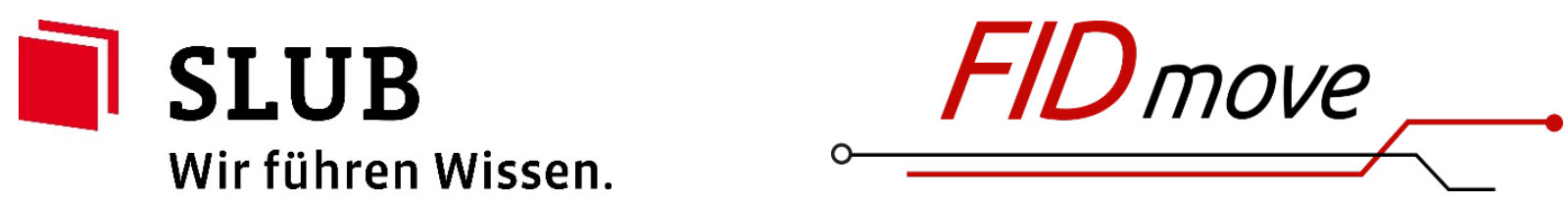


\title{
Conceptual interplanetary space mission design using multi-objective evolutionary optimization and design grammars
}

\author{
A Weber $^{1 *}$, S Fasoulas ${ }^{2}$, and $\mathbf{K}$ Wolf ${ }^{1}$ \\ ${ }^{1}$ Institute for Aerospace Engineering, Technische Universität Dresden, Dresden, Germany \\ ${ }^{2}$ Institute of Space Systems, Universität Stuttgart, Stuttgart, Germany
}

The manuscript was received on 29 October 2010 and was accepted after revision for publication on 28 March 2011.

DOI: $10.1177 / 0954410011407421$

\begin{abstract}
Conceptual design optimization (CDO) is a technique proposed for the structured evaluation of different design concepts. Design grammars provide a flexible modular modelling architecture. The model is generated by a compiler based on predefined components and rules. The rules describe the composition of the model; thus, different models can be optimized by the $\mathrm{CDO}$ in one run. This allows considering a mission design including the mission analysis and the system design. The combination of a CDO approach with a model based on design grammars is shown for the concept study of a near-Earth asteroid mission. The mission objective is to investigate two asteroids of different kinds. The CDO reveals that a mission concept using two identical spacecrafts flying to one target each is better than a mission concept with one spacecraft flying to two asteroids consecutively.
\end{abstract}

Keywords: conceptual design optimization, multi-objective optimization, evolutionary algorithms, design grammar, spacecraft design, near-Earth asteroids

\section{INTRODUCTION}

The usage of optimization techniques in the early development phase of a product is called conceptual design optimization (CDO). Applied to spacecraft design, the main intention is to find possible basic concepts or design solutions which satisfy the mission requirements. For example, a typical question in a conceptual design phase is whether a chemical or an electrical propulsion system is more efficient for this mission.

The advantages of CDO were proven by several authors in recent years. Mosher [1] demonstrated the design of an interplanetary mission like NEARShoemaker using evolutionary algorithms to optimize a top-level model describing the main components of an interplanetary spacecraft. Taylor [2] compared different methods for the optimization of Earth

*Corresponding author: Institute for Aerospace Engineering, Technische Universität Dresden, Dresden 01062, Germany. email:andreas.weber@tu-dresden.de surveillance missions. Jilla [3] applied the method for the optimization of a satellite constellation and Hassan [4] for reliability optimization. These examples are focused on one domain. They do not combine models of different domains like mission analysis and system design. Jilla [3] and Hassan [4] also gave a comprehensive overview of other applications in aerospace engineering. The CDO enables the structured inspection of many different possible design points. It gives an insight into the potential and limitations of solutions. Thus, the CDO provides an understanding of the design space and supports the decision-making process. It is not intended as a substitute of the decision maker. The generated results are proposals among which the decision maker can choose according to the various other design drivers which are not modelled.

The examples reveal the characteristic of CDO:

(a) models used in conceptual design are of different fidelity (rule of thumb, analytical or numerical models); 
(b) models use different kinds of variables (quantitative, qualitative, discrete, continuously);

(c) some quantities for modelling the design implications can be only expressed in qualitative values;

(d) design points typically have different topologies, meaning that the design points are described by different variables.

This implies that special constraints have to be applied to the models used in the CDO approach. A flexible modelling toolbox is needed, which provides modules for different problems. These modules must allow the creation of models according to the specific design problem. The resulting models must be able to handle different kinds of solutions, e.g. a chemical or an electrical propulsion system with different describing variables. Additionally, the models must be transparent and easy to adopt.

Rudolph [5] proposed the usage of a grammar to create models in the design process. Like linguistic grammar, a grammar for design also consists of vocabulary and rules. The rules describe the valid combination of the vocabulary. The vocabulary can represent various kinds of information, including models or submodels. Schaefer and Rudolph [6] already demonstrated the application of design grammars to the design of spacecrafts. Using design grammars means that the model is generated by a compiler based on the vocabulary and the rules. Thus, with different sets of rules, models describing different systems can be created. Design grammars are, therefore, a flexible model framework.

This flexibility can be used to combine a mission analysis model with a system design model of a spacecraft. Integrated in an optimization, various mission concepts can be evaluated in a structured process. This includes trades between the trajectory design and the spacecraft design. Thus, the usage of design grammars for the models in a CDO framework promises serious advantages. In section 2, the CDO method and the design grammar is described. Section 3 gives an example on how the method is applied. The results are presented in section 4 . Finally, section 5 summarizes this study.

\section{CONCEPTUAL DESIGN OPTIMIZATION}

\subsection{Multi-objective evolutionary optimization}

The design can be understood as a search for an optimal solution. A design problem consists of a desired goal, a search space, and a search method [7]. Design optimization problems are typically multi-criteria problems with two or more design goals. These criteria are usually contradictory. A spacecraft, for example, should have a minimal total mass and a high payload mass. Additionally, design optimization problems are described by parameters which are known only qualitatively and those which are known quantitatively. Thus, the design problem consists of a combination of discrete and continuous variables. There might be applicable knowledge about the design problem, but in general, the problem is modelled as a black box.

There are several optimization algorithms available for this kind of multi-objective optimization problem. An overview can be found in Andersson [8]. One common group of methods is evolutionary computing. The name is derived from the imitation of biological evolution. A detailed description can be found in Coello et al. [9]. Evolutionary computing can optimize problems without gradient information.

In the scope of this study, an evolutionary computing tool developed for the optimization of aircraft structures $[\mathbf{1 0}, \mathbf{1 1}]$, called GEOpS (genetic and evolutionary optimization of structures) is used. It applies a combination of three algorithms: genetic algorithm (GA), evolutionary strategies (ES), and differential evolution. Although intended for the optimization of aircraft structures, it uses abstract interfaces capable of optimizing problems in various domains.

In the following paragraphs, the standard methods used in GEOpS are shortly summarized. For more details, refer to Kaletta [10]. For multiobjective optimization, Goldberg's ranking is used, meaning that equal probability of reproduction is assigned to all non-dominated individuals in the population. For selection, fitness proportional selection also known as Roulette-wheel selection is used. The selection strategy is plus, meaning that parent and child population are used to create the new parent population. All applied algorithms use crossover or recombination and mutation as operators. The GA uses a five-point crossover. The ES work with discrete and intermediate recombination. In all crossover methods, two parent individuals are involved.

In each generation, 70 individuals are computed. Typically, about 300 generations are calculated. The computational effort is reduced with two actions. First, the inherent parallel structure of evolutionary computing is used for parallelization. All newly created individuals are available at the same time. This permits the program to evaluate them in parallel. In GEOpS, the message passing interface (MPI) is used to execute the parallel evaluation. MPI represents a standard for parallel computation in multi-processor environments. Therefore, PC-clusters, PCs with 
multi-core CPUs as well as high-performance computers can be employed. Second, the design space is adjusted during the optimization process. In GEOpS, this is achieved by a rather simple approach: the feasible region of the design space is reduced during the optimization run, depending on the parameter range of the best individuals. In order to prevent a premature stagnation of the optimization, a margin of safety is introduced for the new interval.

\subsection{Principle of design grammars}

A design grammar is the definition of a formal language for the description of a design. Formal languages can describe processes or models. In 1968, Lindenmayer already proposed formal languages for the modelling of plants and their growth process [12]. Recently, formal languages were proposed for the usage in the design process and the application was shown for various engineering design domains [5, 13], including satellite design [6]. This section gives a short introduction based on Rudolph [5] and Schaefer and Rudolph [6].

A grammar $G$ consists of a vocabulary $V$, a number of rules $R$ and an axiom $A$. The grammar $G=\langle V, R, A\rangle$ defines the composition of a word list or sentence $V^{*}$ by starting with the axiom $A$ and applying the rules $R$ consecutively. In formal languages, the vocabulary is typically concatenated linearly. In contrast, for engineering design, the vocabulary must be linked in complex networks. Therefore, the sentences formed by design languages are represented by a graph structure called design graph. Nodes in the graph are representing the words; the edges are the links between the words.

The vocabulary represents the description of elements of a design. Elements can be physical parts as well as logical or functional components. For example, physical parts can include information about the dimensions, shape, materials, interfaces, and functionality. These properties can also be connected to models or algorithms providing the ability to calculate values. Properties are divided into input and output properties. The links between nodes represent the exchange of output properties of one node accordingly with the input properties of another node. The rules define operations like adding or deleting a node in the design graph.

For the creation of a design, a design compiler is used to interpret the defined grammar $G$. Starting with the axiom, the design compiler combines the words or changes properties according to the defined rule set. The result is a design graph, which includes all necessary information about the designed system.
In the scope of this study, the design grammar is used to create a model for the design of spacecraft. Thus, the vocabulary consists of models for mission analysis and the design of various components. Using design grammars allows including the structure or topology of the model in the model itself. With the same vocabulary, the final design graph can vary depending on the applied rules [5].

For example, to model a spacecraft having a chemical or an electrical propulsion system, a different rule is applied in each case. This rule change assures that the corresponding node is used. The design compiler assures that the different required inputs for the nodes are fulfilled. The results are two different models, one for a spacecraft with a chemical propulsion system and another for a spacecraft with an electrical system.

\subsection{Optimization concept}

There are two basic concepts for the combination of design grammars with the multi-objective evolutionary optimization. The design compiler can be used once for the generation of the model and in a second step, the model is optimized. This implies that the model structure is not changeable during the optimization. The second concept generates the model in each model evaluation step based on the input variables. For each input variable, combination the model can have another internal structure. This provides high flexibility, but also increases the computational effort. A combination of both approaches is possible. In this case, the generated model needs to be saved and loaded each time a similar model structure is used. This requires that the optimization process has some kind of knowledge about the model structure. Although this might improve the optimization speed, it does not change the principle. For simplicity the second concept is used. As depicted in Fig. 1, the model is generated for each evaluation step.

For each set of design variables, the model provides the fitness values and a set of parameters describing the design. The fitness values are the input for the optimization process. If the stop criterion is not reached, a selection step is started, resulting in a new generation. The individuals of the generation are modified and evaluated. Once again, for each individual, the design compiler is used to generate the model and calculate the fitness and design values. The process is repeated until the stop criterion is fulfilled.

The approach presented in this study allows a multi-objective optimization where the optimized model can handle different types of problems. 


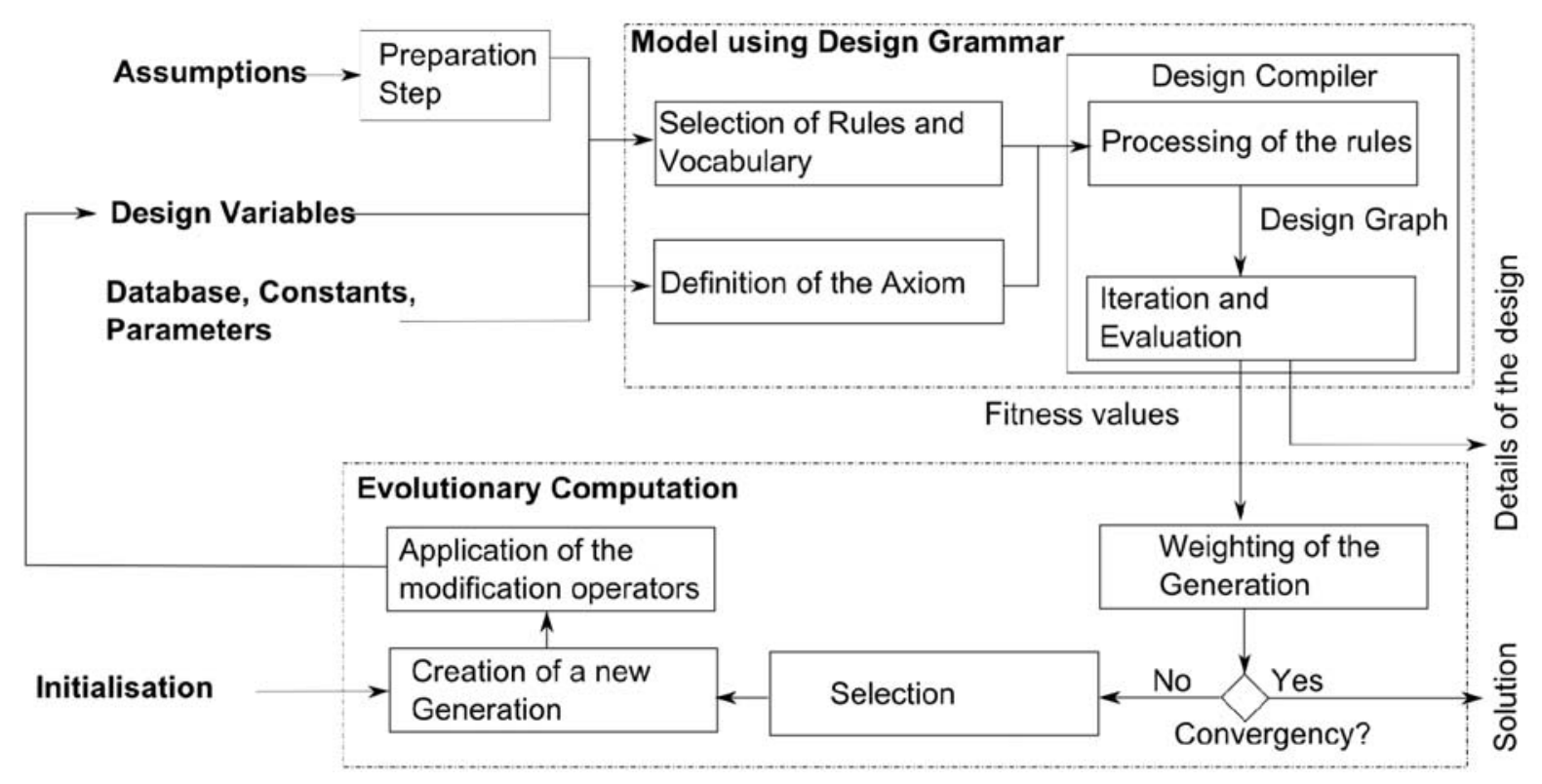

Fig. 1 Concept of the multi-objective optimization using a model based on design grammar and evolutionary computation as optimization tool

\section{MODEL DEVELOPMENT}

\subsection{Motivation}

As an example, a multi-rendezvous exploration mission to near-Earth asteroids is used. The knowledge about near-Earth asteroids so far is very limited. The orbits of more than 6820 near-Earth asteroids are known as of November 2010; about 700 asteroids are characterized with Earth-based observations $[14,15]$. Nonetheless, in situ characterization can substantially improve the knowledge about asteroids. The intention of this example is to show the potential of small satellites for the characterization of multiple asteroids. The mission objective is motivated by recent studies like ASTEX [16]. The objective is to visit two asteroids of different taxonomic types.

The taxonomic type classifies the asteroids according to their spectral shape, colour, and albedo [17]. There are two major types depending on the different mineralogical compositions of the asteroid surface: dark carbonaceous objects (type C) and stony objects (type S). The mission objective is to visit one asteroid of each type. The mission can be fulfilled by one spacecraft flying consecutively to both asteroids or by two identical spacecrafts each flying to one asteroid. These two options are traded against each other.

As a first step, the model is limited to trajectory design, system design, and launcher selection. A cost modelling is not included. It is assumed that the production cost is relatively small, compared to the cost for development and that the development cost scales with the satellite mass. Constraining the multi-spacecraft architecture to two identical spacecrafts is supposed to keep the results comparable to the single spacecraft architecture.

\subsection{Model description}

The design grammar is intended to create a model for the calculation of a basic mission architecture, including the selection of the best launcher, estimation of a transfer trajectory, and a rough mass and power budget of the satellite. The following design variables (input variables which are optimized) are used:

(a) targets;

(b) launch date and interval for time of flight;

(c) number of revolutions for low thrust transfer trajectories;

(d) initial orbit energy C3;

(e) payload mass;

(f) propulsion system type;

(g) mission architecture.

The model provides the following output variables:

(a) spacecraft launch mass $m_{\text {tot }}$ and mass budget of the satellite;

(b) transfer trajectory $r(t)$, including the launch date and the necessary $\Delta v$;

(c) duration of the science phase $t_{\mathrm{sc}}$;

(d) evaluation of the quality of the mission target $b_{\text {comb }}$ based on the scientific relevance of the selected targets;

(e) the smallest possible launcher, represented by the rank $k_{\text {launch. }}$. 
The vocabulary represents models for the mission design. There are 10 different nodes for: trajectory design, propulsion subsystem, power subsystem, overall satellite design, launcher selection, etc. The defined rules allow the generation of four different models. The rules are selected depending on the propulsion system and the mission architecture.

In the case of the propulsion system, there is one node for the chemical and one for the electrical propulsion. They are replaced by one another, based on the provided propulsion system type. The mission architecture selects a rule leading to different model topologies, as depicted in Fig. 2. Case (a) is a mission with one spacecraft flying to two targets consecutively; case (b) uses two identical spacecrafts flying to one target each. Instead of one mission and one transfer node in case (b), two instances of the mission and target nodes are included in the model. The design compiler assures that these additional nodes are connected correctly.

\subsection{Description of the model background}

The information about the targets is retrieved from a database combining information about the orbital elements and known characterization based on Binzel et al. [14] and NASA JPL [15]. The amount of possible target combinations is reduced by selecting all asteroids with a minimum magnitude of 25 and a $\Delta v<8 \mathrm{~km} / \mathrm{s}$. For each of these asteroids, the necessary $\Delta v$ is calculated to reach each of the other asteroids in the lists. There remain about 200 combinations of two asteroids with total $\Delta v$ estimations for the transfer Earth - Asteroid 1 - Asteroid 2 of less than $12 \mathrm{~km} / \mathrm{s}$. The known information about the targets (orbital elements, physical properties like diameter, rotation rate, and taxonomic type) are used to generate a ranking of the scientific and technological relevance $b_{\text {comb }}$.

With the provided launch date and the orbital energy C3, Lambert's problem is solved and in the case of chemical propulsion system, the transfer trajectory is calculated. To reduce the search space, the time of flight is an interval. The model uses a local search to find the optimal time of flight for the given launch date. For low thrust propulsion systems, a trajectory is estimated using a shape-based approach proposed by Wall [18]. The trajectory calculations assume a preliminary mass estimation. Based on the estimated transfer trajectory, the required power budget is calculated, including the specific characteristic of the chosen propulsion system. The model includes the PPS 1350 engine [19] used on SMART-1 and the QinetiQ T5 engine [20] as low thrust propulsion systems.

The power budget and the necessary $\Delta v$ are used to approximate the mass of the propulsion and power subsystems based on rules of thumb [21]. The mass of the structure, the thermal subsystem, and the harness is calculated by a total mass ratio. For the command and data handling, fixed assumptions of the communication and attitude and orbit control subsystems based on similar missions are used. The sum of all partial mass assumptions provides a new launch mass. With the new launch mass and the given C3, the smallest possible launcher is selected. The calculation is repeated until the launch mass converge within a given interval. (a)

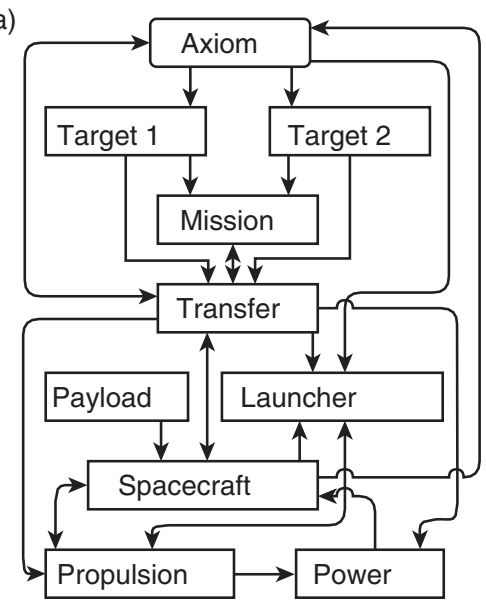

(b)

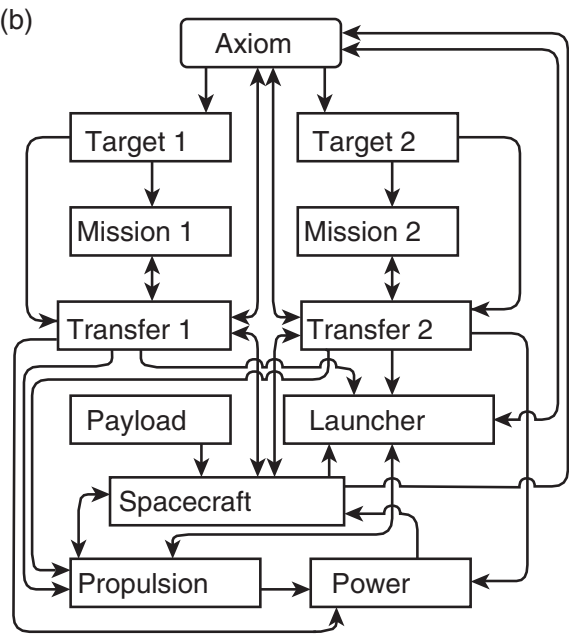

Fig. 2 Structure of the model for: (a) an architecture with one spacecraft flying to both targets consecutively and (b) an architecture with two identical spacecraft flying to one target each Note: The propulsion system can be either chemical or electrical 


\subsection{Model validation}

The validation of the model is done by a comparison with previous missions or mission studies. Based on the $\Delta v$, the input power, the payload mass, and the type of propulsion system, a concept for the different examples is calculated and compared to the original, as depicted in Fig. 3. Five of the seven considered examples have a deviation of less than 20 per cent. In the Simone study [22], novel technology is considered, but not implemented in the model. The deviation in the DonQuijote [23] example indicates that design drivers which had been considered in the study were not implemented in the model.

The comparison proves that the model produces realistic mission concepts. The results are conservative compared to newer missions like Hayabusa [24] or SMART-1 [25].

\subsection{Objective function and optimization}

The number of objectives is limited by the necessary computational effort and the capability for the evaluation of the results. With an increased number of objectives, the number of individuals calculated in each generation also needs to be increased, to reach a similar search density in the solution space. Up to three objective values can be easily visualized. Therefore, the objective function is a three-dimensional vector of weighted sums of the values provided by the model. The first objective value represents the performance of the target combination based on the scientific and technological evaluation $b_{\text {comb }}$ and the length of the science phase $t_{\mathrm{sc}}$. The second objective represents the quality of the spacecraft design. It is the sum of the normalized spacecraft launch mass $m_{\text {tot }}$ and the rank of the launcher system $k_{\text {launch. }}$ The third objective value gives an

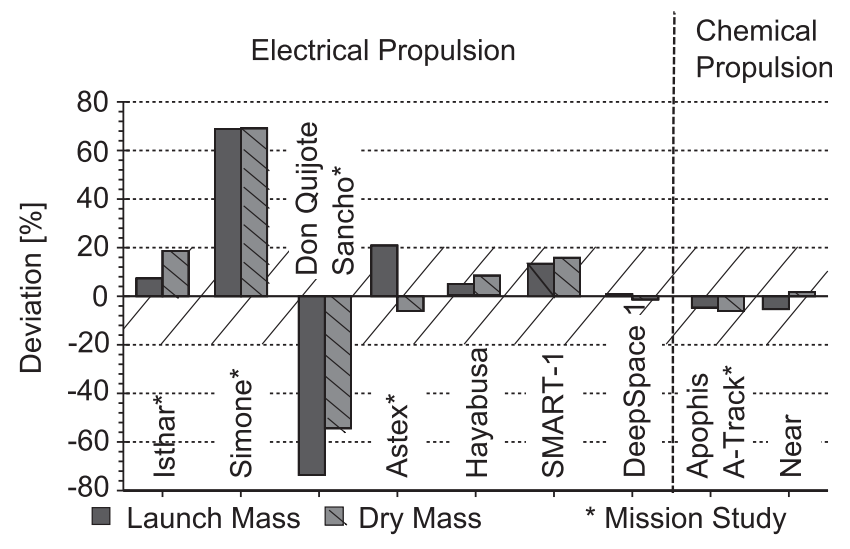

Fig. 3 Comparison of the developed model with missions and mission studies Note: The data for comparison is taken from references [16], [22] to $[\mathbf{2 9}]$ insight into the performance of the transfer problem solution. It is the sum of the necessary normalized $\Delta v$ and the required normalized transfer time. The launch mass, $\Delta v$, and transfer time are normalized by their maximal allowable value.

This grouping of the performance evaluations allows the trading of spacecraft mass and launcher system as a measure for mission cost against the scientific relevance. It is necessary to include the solution of the transfer problem, as done with the third objective function, to guide the optimizer.

Due to the stochastic nature of the problem, the optimization is repeated 10 times and the results are combined into an overall solution. The computational effort for one individual is about $2 \mathrm{~min}$ on a standard desktop PC. With about 300 generations per run and 70 individuals per generation more than 21000 model evaluations are needed. This results in rather long optimization runs. The main reason is a slow implementation of the low thrust transfer model.

\section{RESULTS}

The result of the optimization runs are a collection of solutions. A part of the collection is pareto-optimal. There are two approaches for evaluating the results. The classic optimization approach would drop all non-pareto-optimal solutions and an engineer can select one or more of the remaining solutions for further investigations. This is usually only feasible if the number of solutions is small. Dropping all nonpareto-optimal solutions also means that suboptimal but maybe good engineering solutions are dropped.

Another approach formalizes the selection by introducing an evaluation step. This evaluation step weights the objectives and returns a ranking. As this is done after the optimization run, the weighting can be changed without the need for running the optimization. With the definition of a preference weighting $\left(w_{1}=0.3 ; w_{2}=0.5 ; w_{3}=0.2\right)$ for the three objectives, a mission with the targets $1999 \mathrm{YB}$ and 1999 RQ36 using two identical spacecrafts with low-thrust propulsion is found as best solution. Table 1 presents an overview of the mission concept.

With the representation of the results in the design space, it is possible to support the engineering process and generate a basic understanding of the problem considered. Typically, an unknown or uncertain design parameter is used to represent the solution. In Fig. 4, the solutions are represented as $m_{\text {tot }}=f(\Delta v)$. As can be seen, there are various possible concepts with a launch mass of about $500 \mathrm{~kg}$, thus in the area of a small satellite mission. In case of changing requirements, the information can be used to get 
an impression of the consequences of design changes.

For the obtained result, in case, the proposed Ariane 5 piggyback launch is not possible or not available, an alternative could be a launch with the Dnepr launcher. This would require an additional kick stage, but the launch mass of the satellite itself would remain. An alternative mission target with similar requirements for the spacecraft could be 1999 NW2 and 2001 QC34.

As indicated in Fig. 4, the single target, electrical propulsion concept is a baseline with various targets of high scientific relevance. A multi-target approach with one spacecraft flying to two targets consecutively is possible, but the target combinations are less preferable. Thus, for the used evaluation scheme of the scientific relevance, a multi-target approach results in non-optimal solutions.

The presented example used a fixed payload mass assumption. With the presented model, the payload mass can be included as a design variable in the optimization. The results then include additional information about the payload mass. The mean payloadto-mass-ratio of a single-target mission with electrical propulsion is $\mu_{\mathrm{pl}}=0.12$. A spacecraft with a dry mass of $500 \mathrm{~kg}$ can have a payload mass of up to $60 \mathrm{~kg}$.

As already mentioned in section 3.1, a cost modelling is not included. Thus, there is no guarantee that the best solution found is also the solution with the smallest required budget. Nonetheless, with the assumptions that:

(a) the development cost scale with the satellite mass;

(b) the production costs are small, compared to the launch cost and the development cost;

(c) a piggyback launch is more than a factor of two cheaper than a standard launch with Soyuz or Ariane 5; (d) the differences in mission operation costs for the concepts are small compared to the development and launch cost;

the results are still comparable. A solution with a small total mass and cheap launcher option will also have a small total cost. The best found solution might not be the cheapest but is still a low-cost mission due to the small satellite mass and the cheap launch option.

\section{CONCLUSION AND OUTLOOK}

The CDO allows an insight into the characteristic of the considered problem. Multiple possible solutions are presented, enabling the decision maker to choose between various alternatives. The design grammar is a prerequisite for CDO of spacecrafts on a system level. Models with design grammars can calculate the performance of different architectures, where each architecture requires a different kind or number of input or output variables. This enables the combination of mission analysis, system design, and launcher selection in one model. The model is constructed out of well-defined small parts, which are easy to verify and to maintain.

The example illustrates that CDO process can benefit from a flexible model environment provided by design grammars. By changing the rule definition the same model can be applied to different problems. In the presented example, both missions to one asteroid use the same spacecraft. With a change in the rules, the design grammar would also allow to model a case with two different spacecrafts. In the same way, an extension of the example to three or even more targets is possible. The set of vocabulary remains the same; only the description of the way how the vocabulary is connected changes.

Table 1 Description of the best solution found with the CDO approach

\begin{tabular}{|c|c|c|}
\hline Target & $1999 \mathrm{YB}$ & 1999 RQ36 \\
\hline Type & S & $\mathrm{C}$ \\
\hline Diameter & $0.6 \mathrm{~km}$ & $0.2 \mathrm{~km}$ \\
\hline Period & - & $4 \mathrm{~h}$ \\
\hline Arrival date & 09.10 .2018 & 30.06 .2018 \\
\hline Flight time (days) & 568 & 450 \\
\hline Launcher & Ariane 5 piggyback, two launches & \\
\hline Launch mass (kg) & 490 & \\
\hline Dry mass (kg), (including $20 \%$ system margin) & 366 & \\
\hline Payload (kg) & 25 & \\
\hline Power $(\mathrm{kg})$ & 66 & \\
\hline Propulsion (kg) & 55 & \\
\hline Structure and thermal (kg) & 74 & \\
\hline \multicolumn{3}{|l|}{ Communication $38 \mathrm{~kg}$, AOCS $32 \mathrm{~kg}$, Flight computer $15 \mathrm{~kg}$} \\
\hline Power $(\mathrm{W})$ & 1070 & \\
\hline
\end{tabular}

Note: AOCS, attitude and orbit control subsystem.Physical properties are taken from reference [14]. 
a) Target combinations orded by objective 1

\begin{tabular}{|c|c|}
\hline $\begin{array}{l}2001 \text { QC34 - } 2000 \text { RW37 } \\
2002 \text { DQ3 - } 2000 \text { RW37 } \\
1999 \text { RQ36 - } 2001 \text { QC34 } \\
2001 \text { AE2 - } 2001 \text { QC34 } \\
1999 \text { YB - 1999 RQ36 } \\
1999 \text { NW2 - } 2001 \text { QC34 } \\
2000 \text { AE205 - } 1999 \text { RQ36 } \\
1999 \text { JU3 - } 1993 \text { VA } \\
2006 \text { HW50 - 3361 Orpheus } \\
2006 \text { CL9 - } 3361 \text { Orpheus }\end{array}$ & 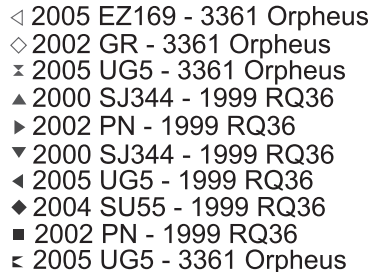 \\
\hline
\end{tabular}

b) Solutions with szenarios marked

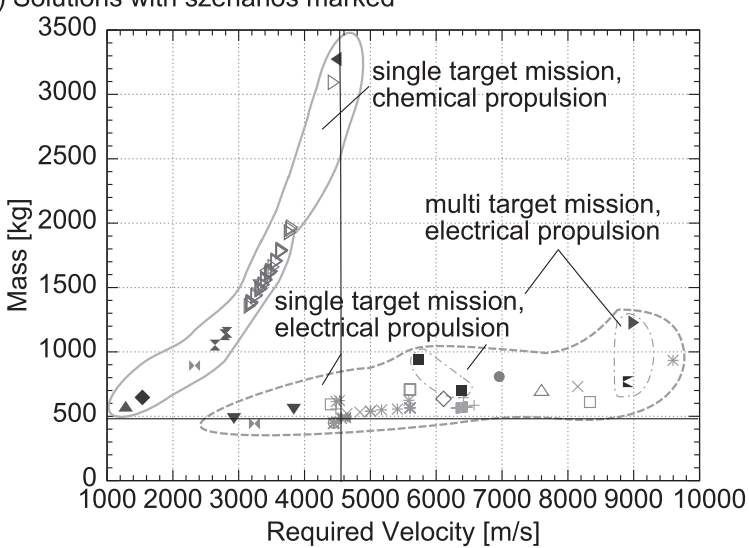

c) Solutions with launcher marked

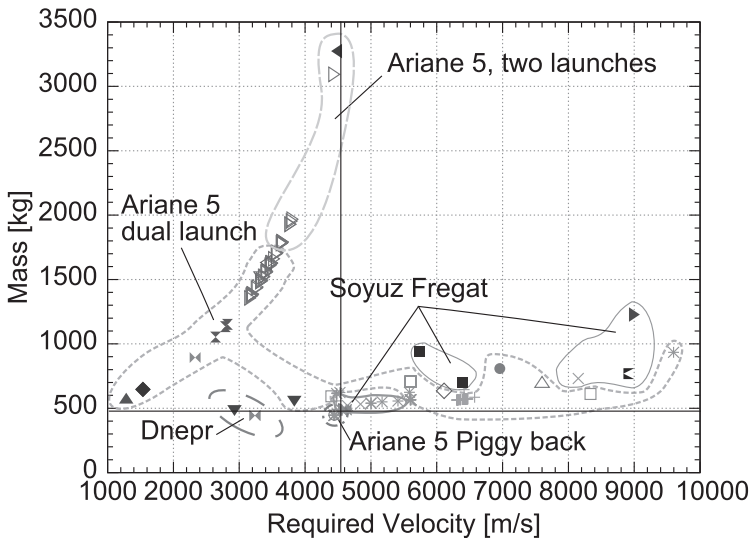

Fig. 4 Results of the CDO

Note: Each point represents a possible mission design. The selected best solution is marked by the crossing lines

The application to the asteroid mission design example shows that exploring different asteroids is more efficient with multiple small spacecrafts flying to one target each than with one spacecraft flying to multiple targets consecutively. Multiple targets restrict the mission design due to the transfer problem boundary conditions like maximum overall mission time and minimum and maximum stay time at the first asteroid. Therefore, only a small number of solutions to less preferable targets were found.

The applied model for the evaluation of interplanetary missions is a first assessment with a focus on preliminary trajectory design and the sizing of the propulsion and power subsystem. A total cost assumption is not yet included. Possible extensions are the inclusion of different design philosophies, reliability levels, or assumptions about mission risks. A cost and a reliability modelling would increase the maturity of the model; both are major design drivers which are not yet considered directly in the model. Further, the model can be improved by a more detailed modelling of the various subsystems.

The method can also be applied to other interplanetary mission concepts. However, up to now, there are two major limitations, due to the modelling tailored to near-Earth asteroids.

1. Gravity-assist manoeuvre is not yet included in the mission analysis but might be essential for the evaluation of mission to targets father away from Earth like Jupiter.

2. The system design is limited to standard solar cells as main power source. Other power sources might be more reliable for other interplanetary missions.

Thus, the state of development with some minor changes would allow the modelling of interplanetary small satellite mission to targets in the inner solar system like Venus, Mars, and the main belt asteroids. For other mission targets, the same method can be applied, but an extension of the models is necessary.

\section{ACKNOWLEDGEMENTS}

The authors thank A. Rathke, Astrium GmbH, for the inspiring comments and information about typical mission scenarios and design restrictions.

\section{FUNDING}

This study was supported in part by Astrium $\mathrm{GmbH}$ Friedrichshafen, Germany.

(C) Authors 2011

\section{REFERENCES}

1 Mosher, T. J. Improving spacecraft design using a multidisciplinary design optimization methodology. Dissertation, University of Colorado, 2000.

2 Taylor, E. R. Evaluation of multidisciplinary design optimization techniques as applied to spacecraft design. In Proceedings of the IEEE Aerospace Conference (Eds) Prophet, R. A., Wright, R. P., Woerner, D., IEEE, Big Sky, Montana, USA, 2000, http://dx.doi.org/10.1109/AERO.2000.879413. 2000, pp. 371-384.

3 Jilla, C. D. A Multiobjective, multidisciplinary design optimization methodology for the conceptual design of distributed satellite systems. Dissertation, Massachusetts Institute of Technology, 2002. 
4 Hassan, R. A. Genetic algorithm approaches for conceptual design of spacecraft systems including multiobjective optimization and design under uncertainty. Dissertation, Purdue University, 2004.

5 Rudolph, S. Übertragung von Ähnlichkeitsbegriffen, 2002 (Habilitation, Universität Stuttgart).

6 Schaefer, J. and Rudolph, S. Satellitenentwurf mit Entwurfsgrammatiken. Aero. Sci. Tech., 2005, 9, 81-91.

7 Renner, G. and Ekárt, A. Genetic algorithms in computer aided design. Comput. Aided Des., 2003, 35(8), 709-726. http://dx.doi.org/10.1016/S0010-4485(03)0 0003-4.

8 Andersson, J. A survey of multiobjective optimization in engineering design. Department of Mechanical Engineering, Linköping University, Version: 2000. http:/ / citeseerx.ist.psu.edu/viewdoc/ summary?doi=10.1.1.8.5638. 2000, Technical Report LiTH-IKPR-1097).

9 Coello, C. A. C., Lamont, G. B., and Veldhuizen, D. A. Evolutionary algorithms for solving multi-objective problems, 2nd ed., 2007 (Springer, New York).

10 Kaletta, P. Ein Beitrag zur Effizienzsteigerung Evolutionárer Algorithmen zur optimalen Auslegung von Faserverbundstrukturen im Flugzeugbau. Dissertationen, Verlag, Cuvillier, Göttingen, Germany, 2006.

11 Seeger, J. and Wolf, K. Structural optimization of adaptive airfoils using evolutionary algorithms. In Proceedings of the 1st CEAS European air and space conference, Germany, CEAS-2007-236.

12 Prusinkiewicz, P. and Lindenmayer, A. The algorithmic beauty of plants, 1996 (Berlin, Springer).

13 Alber, R. Synthese und Evolution einer technischen Entwurfsgrammatik nach dem Vorbild biologischer Wachstums- und Entwicklungsprinzipien. Thesis, Institut für Statik und Dynamik der Luft- und Raumfahrtkonstruktionen, Universität Stuttgart, January 2001.

14 Binzel, R. P., Lupishko, D. F., Dimartino, M., Whiteley, R. J., and Hahn, G. J. Physical properties of NEOs. In Asteroids III (Eds W. F. Bottke, P. Paolicchi, R. P. Binzel, A. Cellino), 2002, pp. 255-271 (University of Arizona Press, Tucson, USA).

15 NASA JPL, Near-Earth Object Program Office: Near Earth objects Database. Internet. http://neo.jpl.na sa.gov. accessed 04.05.2008.

16 Nathues, A., Boehnhardt, H., Harris, A. W., Jentsch, C., Schaeff, S., Weischede, F., Wiegand, A., Schmitz, N., Goetz, W., and Kachri, Z. ASTEX: An in situ exploration mission to two near-Earth asteroids. $A d v$. Space Res., 2010, 45(1), 169-182. http://dx.doi.org/ 10.1016/j.asr.2009.10.008.

17 Bus, S. J., Vilas, F., and Barucci, M. A. Visible-wavelength spectroscopy of asteroids. In Asteroids III (Eds W. F. Bottke, A. Cellino, P. Paolicchi, R. P. Binzel), 2002, p. 169 (University of Arizona Press, Tucson, USA).

18 Wall, B. J. Technology for the solution of hybrid optimal control problems in astronoutics. Dissertation, University of Illinois, USA, 2007.

19 Rathsman, P., Kugelberg, J., Bodin, P., Racca, G. D., Foing, B., and Stagnaro, L. SMART-1: Development and lessons learnt. Acta Astronaut. 2005, 57(2-8), 455-468. http://dx.doi.org/10.1016/j.actaastro.2005. 03.041. Infinite Possibilities Global Realities, Selected Proceedings of the 55th International Astronautical Federation Congress, Vancouver, Canada, 4-8 October 2004.

20 Edwards, C. H., Wallace, N. C., Tato, C., and Van put, P. The T5 ion propulsion assembly for drag compensation on GOCE. In Proceedings of the Second International GOCE user workshop 'GOCE, The geoid and oceanography', Frascati, Italy, ESAESRIN.

21 Larson Hrsg, W. J. and Wertz Hrsg, J. R. Space mission analysis and design, 3rd ed., 2005 (Microcosm Press).

22 Wells, N., Walker, R., Green, S., and Ball, A. SIMONE: Interplanetary microsatellites for NEO rendezvous missions. Acta Astronaut. 2006, 59(8-11), 700-709. http://dx.doi.org/10.1016/ j.actaastro.2005.07.036. In Selected Proceedings of the Fifth IAA International Conference on Low cost planetary missions.

23 Milani, A., Valsecchi, G., Paolicchi, P., Lognonné, P., Benz, W., Foerstner, R., Bello, M., and Gonzalez J. A. Near Earth objects space mission preparation: Don Quijote mission executive summary. DEIMOS Space S.L. Version: 2003. http://www. esa.int/gsp/completed/neo/donquijote_execsum.pdf. 2003.

24 Uesugi, K. T. Space engineering spacecraft (MUSES) program in ISAS featuring its latest mission, $H A Y A B U S A$. In Proceedings of the International Conference on Recent Advances in Space Technologies, Japan, 20-22 November. 2003, pp. 464-471.

25 Rathsman, P., Kugelberg, J., Bodin, P., Racca, G. D., Foing, B., and Stagnaro, L. SMART-1: Development and lessons learnt. Acta Astronaut., 57. Infinite possibilities global realities, Selected Proceedings of the 55th International Astronautical Federation Congress, Vancouver, Canada, 2005, pp. 455-468. http://dx.doi.org/10.1016/j.actaastro. 2005.03.041 4-8 October 2004.

26 D'Arrigo, P. The ISHTAR mission - executive summary. Astrium Ltd. Version: 2003. http://www. esa.int/gsp/completed/neo/ishtar_execsum.pdf. 2003.

27 Santo A. G., Lee S. C., and Gold R. E. NEAR spacecraft and instrumentation. The Johns Hopkins University Applied Physics Laboratory. Version: 1999. http://near.jhuapl.edu/PDF/SC_Inst.pdf. 1999, accessed 15.01.2010.

28 DEIMOS space: planetary society's apophis mission design competition a-track mission proposal. DEIMOS Space S.L. Version: 2007. http://planetary. s3.amazonaws.com/projects/apophis_competition/ apophis_winner_atrack.pdf. 2007, accessed 07.04.2010.

29 Rayman, M. D. and Lehman, D. H. Deep space one: NASA's first deep-space technology validation mission. Acta Astronaut., 1997, 41(4-10), 289-299. http://dx.doi.org/10.1016/S0094-5765(98)00073-3. 